\title{
Rheological properties of nanocellulose suspensions: effects of fibril/particle dimensions and surface characteristics
}

\author{
Tobias Moberg $\mathbb{D} \cdot$ Karin Sahlin $\cdot$ Kun Yao $\cdot$ Shiyu Geng • \\ Gunnar Westman $\cdot$ Qi Zhou $\cdot$ Kristiina Oksman $\cdot$ Mikael Rigdahl
}

Received: 29 November 2016/Accepted: 28 March 2017/Published online: 12 April 2017

(C) The Author(s) 2017. This article is an open access publication

\begin{abstract}
The rheological properties of aqueous suspensions based on three different nanocelluloses were compared. One system was obtained via acid hydrolysis (thus yielding crystalline nanocellulose, $\mathrm{CNC}$ ) and the other two from mechanical shearing, but from different origins and subjected to different pretreatments. Of the latter two, one was considered to be a rather typical cellulose nanofibril (CNF) suspension whereas the other was a kind of intermediate between CNF and CNC. All three nanocellulose elements differed in dimensions as evident from
\end{abstract}

T. Moberg $(\bowtie) \cdot$ M. Rigdahl

Department of Materials and Manufacturing Technology, Chalmers University of Technology, 41296 Gothenburg, Sweden

e-mail: tobias.moberg@chalmers.se

K. Sahlin · G. Westman

Department of Chemistry and Chemical Engineering, Chalmers University of Technology, 41296 Gothenburg, Sweden

K. Yao · Q. Zhou

School of Biotechnology, Royal Institute of Technology, 10044 Stockholm, Sweden

S. Geng $\cdot$ K. Oksman

Division of Materials Science, Luleå University of

Technology, 97187 Luleå, Sweden

T. Moberg - K. Sahlin · K. Yao · S. Geng ·

G. Westman · Q. Zhou · K. Oksman · M. Rigdahl Wallenberg Wood Science Center, Royal Institute of Technology, 10044 Stockholm, Sweden transmission electron microscopy and atomic force microscopy. With regard to the length of the fibrils/particles, the three nanocelluloses formed three distinct groups with lengths between 200 and slightly more than $800 \mathrm{~nm}$. The three cellulosic elements were also subjected to a TEMPO-mediated oxidation yielding a similar carboxylate content in the three systems. Furthermore, the TEMPO-oxidized elements were grafted with poly(ethylene glycol) (PEG). The amount of grafted PEG was about $35 \mathrm{wt} \%$. The shear viscosity, the storage modulus and the loss modulus of suspensions of the unmodified, the TEMPO-oxidized and the grafted nanocelluloses were determined at room temperature and the solids content of the suspensions was varied between 0.7 and $2.0 \mathrm{wt} \%$. It was concluded that the rheological properties varied significantly between the suspensions depending on the dimensions of the cellulosic elements and their surface characteristics. In this context, the length (or the aspect ratio) of the particles played a very important role.

Keywords Cellulose nanocrystals $\cdot$ Cellulose nanofibrils $\cdot$ Rheology $\cdot$ Surface characteristics

\section{Introduction}

Nanocellulose can be said to be cellulose particles/fibrils which have a width or diameter smaller than 
$100 \mathrm{~nm}$. Often the nanocellulose materials are further subdivided into three different categories (Eichhorn et al. 2009); fibrillated cellulose which consists of long flexible fibrils manufactured using e.g. mechanical shearing, nanocrystals or whiskers which are shorter rod-like particles, the last group being bacterial cellulose (which is produced by bacteria). With regard to the shape or geometry, the elements from the latter group are rather similar to fibrillated cellulose. From an industrial perspective, the two first groups are likely to be of greater importance since the corresponding manufacturing processes are potentially easier to scale-up than the production of bacterial cellulose.

Cellulose nanofibrils (denoted CNF), as the fibrillated nanoparticles are often called, are primarily manufactured using different forms of mechanical shearing of wood fibres. It was first reported on by Turbak and coworkers in the early eighties (Turbak et al. 1983), but the progress was partly hampered due to the excessive energy requirements for shearing the fibres into fibrils. This drawback was to a significant extent alleviated by the use of chemical pre-treatments that substantially lowered the required energy input. Nowadays several different such treatments has been reported on in the literature, some examples are enzymatic treatments (Henriksson et al. 2007), carboxymethylation (Wågberg et al. 2008) and TEMPOmediated oxidation (Isogai et al. 2011). These pretreatments do not only lower the energy requirement for the mechanical shearing but they also change the surface properties of the material. However, advances in the grinding technology may also give positive effects which regard to the energy requirement, cf (Wang and Zhu 2016).

Cellulose nanocrystals (CNC) are obtained by acid hydrolysis of cellulosic fibers/fibrils, most often using hydrochloric acid or sulphuric acid, cf e.g.(Hasani et al. 2008). The treatment dissolves the amorphous parts of the cellulose and leaves short rod-like nanoparticles usually referred to as CNC. Using e.g. sulphuric acid for the hydrolysis leaves sulphate groups on the surface (Jiang et al. 2010). Hence, this kind of treatment also changes the surface of the native cellulose.

The interest in $\mathrm{CNF}$ and $\mathrm{CNC}$ can to a significant extent be attributed to their potentially outstanding mechanical properties (in the first hand the stiffness), which, for example, makes these materials interesting as reinforcing elements in polymer composite materials. Values of the tensile modulus between 100 and $160 \mathrm{GPa}$ have been mentioned (Sakurada et al. 1962; Lee et al. 2014), which is of the same order as the modulus of aramid fibres. The incorporation of $\mathrm{CNF} / \mathrm{CNC}$ into polymer matrices is however hampered by that these cellulose materials normally are available in the form of water suspensions of rather low concentrations.

With regard to use of $\mathrm{CNF} / \mathrm{CNC}$-containing products, processing issues relating to coating, extrusion, moulding etc. come into focus. Here the rheological properties of the nanocellulose suspensions are of prime concern and there are several studies on this subject. In general, suspensions based on CNF/CNC exhibit a complex rheological behaviour; already at low concentrations of the cellulose material. They are shear-thinning, viscoelastic and exhibit an extensional viscosity, cf (Pääkkö et al. 2007; Iotti et al. 2010; Karppinen et al. 2011; Moberg and Rigdahl 2012; Naderi et al. 2014; Moberg et al. 2014). It is also known that different types of additives as well as the concentration can have a significant influence on the rheological character of the suspensions, cf e.g. Karppinen et al. (2011) and Iotti et al. (2010). In many of the studies on the rheological behaviour of nanocellulose suspensions only one type of CNF or $\mathrm{CNC}$ has been employed. The present study will focus on the effect on the rheological behaviour of suspensions of $\mathrm{CNF} / \mathrm{CNC}$ having different dimensions and surface character of the cellulosic elements. Here, however, it should be noted that Naderi and Lindström (2016) compared the rheological behaviour of three CNF-suspensions based on enzymatically treated, carboxymethylated and carboxymethyl cellulose grafted cellulose and they concluded that the CNFsystems could exhibit completely different rheological properties.

In the present work, the rheological properties of aqueous suspensions based on three different nanocelluloses are compared. One system was obtained via acid hydrolysis (thus yielding CNC) and the other two from mechanical shearing, but from different origins and subjected to different pretreatments. Of the latter two, one was considered to be a rather typical CNF suspension whereas the other was a kind of intermediate between $\mathrm{CNF}$ and $\mathrm{CNC}$. All three nanocellulose elements differed in dimensions as evident from the performed analysis using transmission electron microscopy and atomic force microscopy. In an attempt to 
obtain a more detailed understanding of how the structural character affected their rheological behaviour, the nanocelluloses were subjected to TEMPO (2,2,6,6-tetramethylpiperidine-1-oxyl)-mediated oxidation in order to contain a similar total carboxylate content. The charging of the cellulosic elements was here assumed to retard the tendency for aggregation and thus have a clear influence on the rheological properties. If the different cellulosic elements had a similar surface charge character, this also in principle allows for more precise evaluation of the effect on the dimensions of the cellulosic elements on the rheological behaviour of the suspensions. In this study, the interaction between the cellulose fibrils/particles were further modified by grafting of poly(ethylene glycol) onto the TEMPO-oxidized samples using a procedure described by Tang et al. (2015). A grafting of this type is of interest in connection to reinforcement of thermoplastics, i.e. for manufacturing of composites, cf (Xu et al. 2013; Moberg et al. 2016). The rheological behaviour of the suspensions based on the original nanocelluloses as well as the treated ones were evaluated in terms of the shear viscosity and the viscoelastic properties, expressed as the form of the storage and loss moduli. The overall conclusion is that the rheological properties of these suspensions differed substantially which points to the importance of a careful characterisation of dimensions and surface character of the elements when comparing such systems and using them in different situations.

\section{Materials}

Three different nanocellulosic materials were used in this work. These were all of different origins and produced following alternative routes resulting in distinctly different shapes of the fibrillar elements and also surface characteristics. These was denoted cellulose nanofibrils (CNF), cellulose nanocrystals obtained from cellulosic material produced by following a bioethanol processing route (BE-CNC) and cellulose nanocrystals obtained from microcrystalline cellulose (CNC). The preparation of the nanocellulosic materials is described in some detail below. Parts of the different cellulosic elements were also subjected to TEMPO-mediated oxidation and subsequent grafting with poly(ethylene glycol) (PEG) as indicated below.

\section{$\mathrm{CNF}$}

The CNF were produced from TEMPO-mediated oxidation of never-dried softwood sulphite pulp followed by mechanical disintegration of the corresponding aqueous suspension as described by Tang et al. (2015). A part of this suspension was grafted with PEG, cf also (Tang et al. 2015; Moberg et al. 2016). The notation TO-CNF will in most cases be used in the following to underline that the fibrils were produced in this manner.

\section{BE-CNC}

The preparation of the BE-CNC is described in detail by Mathew et al. (2014). The starting material was unbarked wood chips from Norwegian spruce. An acid pre-treatment of the chips in a bioethanol pilot plant gave a solid cellulose-containing residue which after further processing steps was disintegrated in a highpressure homogenizer yielding a $2 \mathrm{wt} \%$ aqueous CNC-suspension. In a series of experiments, the produced BE-CNC was also subjected to the TEMPO-mediated oxidation performed in a similar way as for the CNF (Tang et al. 2015) in order to facilitate a comparison between the rheological behaviours of the suspensions. Some of the oxidised sample was then subjected to grafting with PEG.

\section{$\mathrm{CNC}$}

CNC suspensions were prepared from microcrystalline cellulose by a sulphuric acid treatment followed by desulfation procedure as described by Kantor and Schubert (1957), Jiang et al. (2010), Hasani et al. (2008). Also in this case, some part of the produced $\mathrm{CNC}$ was subjected to the TEMPO-mediated oxidation at similar conditions as the $\mathrm{CNF}$ and a subsequent PEG-grafting in the same way as for the BE-CNC.

Surface modification of the cellulosic nanomaterials

As stated above, both the BE-CNC and the $\mathrm{CNC}$ were TEMPO-oxidised following the route outlined in Tang et al. (2015). The reasons for this can be said to be twofold. Firstly, through this treatment the surface charge of the different cellulosic elements became rather equal, facilitating the interpretation of the 
rheological properties in terms of structural differences between the three nanocellulosics. Secondly, it enabled grafting of amino-terminated PEG via coupling of the amine group to the carboxyl group resulting from the oxidation of the C6 primary hydroxyl on the surface of the cellulosic elements from the TEMPO-oxidation (Tang et al. 2015).

All the three nanocellulosics (CNF, BE-CNC and CNC) were grafted with amino-terminated PEG (PEG$\mathrm{NH}_{2}$ with $\bar{M}_{w}=750 \mathrm{~g} \mathrm{~mol}^{-1}$ ) using the procedure described by Tang et al. (2015). As already mentioned, $\mathrm{BE}-\mathrm{CNC}$ and $\mathrm{CNC}$ were TEMPO-oxidised before the grafting. PEG- $\mathrm{NH}_{2}$ was here synthesized according to a previously reported method (Mongondry et al. 2003). The target for the amount of grafted PEG on the cellulosic elements was around $35 \mathrm{wt} \%$ (relative to the mass of cellulose), see also later in the text.

\section{Characterization methods}

The carboxylate content of CNF, BE-CNC and CNC (including the TEMPO-oxidised samples) as well as the content of residual carboxyl groups in these samples after grafting, was determined by conductometric titration. For the conductometric titration, typically, 100-300 mg sample suspensions were used and the $\mathrm{pH}$ of the suspensions was adjusted to 3.0 with $0.01 \mathrm{M} \mathrm{HCl}$. The suspension was titrated with $0.05 \mathrm{M}$ standardized $\mathrm{NaOH}$ by adding $0.1 \mathrm{~mL}$ aliquots in $60 \mathrm{~s}$ intervals until the $\mathrm{pH}$ value reached 11 , and the conductivity was monitored with a conductometric station (SevenCompact, Mettler-Toledo).

Fourier transform infrared spectroscopy (FTIR) of the samples was performed using a Perkin-Elmer Spectrum 2000 FTIR equipped with a MKII Golden Gate, single reflection attenuated total reflectance (ATR) system from Specac Ltd., London, UK. The ATR crystal was a MKII heated diamond $45^{\circ}$ ATR top plate and the samples were obtained from freeze drying of the aqueous suspensions. The spectral range of the measurements was $600-4000 \mathrm{~cm}^{-1}$ with a resolution of $4 \mathrm{~cm}^{-1}$.

The morphology of samples (or more specifically the dimensions of the fibrils and the CNC-particles) was examined both by transmission electron microscopy (TEM) and atomic force microscopy (AFM). For the TEM measurement a drop of the dilute water suspension of nanocellulose was deposited on a carbon-coated grid and treated with $1 \%$ uranyl acetate negative stain. The instrument used was a Hitachi Model HT7700 transmission electron microscope operating in high-resolution mode at $100 \mathrm{kV}$. The length and diameter of individual fibrils (TO-CNF) and BE-CNC entities were assessed from the TEM micrographs using a software procedure described by Usov and Mezzenga (2015). For the AFM measurements, a dilute suspension $\left(10^{-4} \mathrm{wt} \%\right.$ in case of the $\mathrm{CNC}$ and the BE-CNC and $10^{-3} \mathrm{wt} \%$ for the TO$\mathrm{CNF}$ ) was placed on a mica surface, and allowed to dry at room temperature before being imaged in the AFM. The AFM analysis was performed in the tapping mode with a Veeco Multimode Scanning Probe (Santa Barbara, USA) with a Nanoscope V software to obtain height and amplitude information of individual cellulosic elements. With CNC, the length of the crystalline elements was evaluated with same software as with the TEM-analysis, whereas for the diameter, the height of the CNC entities was used (in order to avoid broadening effects) as outlined by Hooshmand et al. (2014). The average values of the dimensions of the elements and their standard deviations were based on measurements of about 200 fibrils/particles (both for TEM and AFM). The AFM was also used in order to obtain representative images of all the used cellulosic elements.

Thermogravimetric analysis (TGA) of the samples was performed with a Mettler Toledo TGA/DSC 1 STARe System using nitrogen as the purge gas at a flow rate of $50 \mathrm{~mL} \mathrm{~min}{ }^{-1}$. The weight of the samples was about $7 \mathrm{mg}$ and they were heated from 30 to $700{ }^{\circ} \mathrm{C}$ at a rate of $10{ }^{\circ} \mathrm{C} \mathrm{min}^{-1}$. TGA was in the first hand used in order to estimate the amount of PEG grafted onto the cellulose surfaces.

The rheological properties of the suspensions was measured at $25{ }^{\circ} \mathrm{C}$ with an Anton Paar MCR 702 rheometer (Graz, Austria) using a cone-plate configuration (cone diameter $50 \mathrm{~mm}$ and cone-plate angle $1.991^{\circ}$ ). For each of the nanocellulosic suspensions, two concentrations were in most cases employed. Both the shear viscosity and the dynamic shear properties, given by the storage modulus $G^{\prime}$ and the loss modulus $G^{\prime \prime}$, were determined. The steady state shear viscosity was measured at shear rates between 0.1 and $100 \mathrm{~s}^{-1}$. The dynamic properties were measured during a strain sweep at $1 \mathrm{~Hz}$ in which the moduli $G^{\prime}$ and $G^{\prime \prime}$ were measured as functions of the applied shear strain amplitude. For each sample, the measurements were, 
in most cases, repeated three times (using different specimens) and the variation in shear viscosity and storage modulus between the specimens was less than $15 \%$ (standard deviation).

\section{Results}

Thermogravimetric analysis (TGA) and degree of grafting

Figure 1 shows the results from the TGA-measurements in the case of TO-CNF and BE-CNC (including their TEMPO-oxidized and grafted counterparts). The degradation curve for pure PEG is also shown and it is obvious that the main decomposition of PEG takes place at a higher temperature (around $400{ }^{\circ} \mathrm{C}$ ) than that of the cellulosic materials. Assuming that the grafted PEG degrades in the same manner as the pure, the amount of grafted PEG can be calculated from the amount of material at the inflexion point in degradation curves for the grafted nanomaterials at the temperature corresponding to the onset of the PEGdegradation as described by Tang et al. (2015). The amount of grafted PEG was $33 \mathrm{wt} \%$ in case of CNF, $38 \mathrm{wt} \%$ for BE-CNC and $39 \mathrm{wt} \%$ with CNC.

In general, the degradation pattern for the different nanocellulosics was quite similar, with an onset of the decomposition around $225^{\circ} \mathrm{C}$. It seemed like the BECNC exhibited a somewhat higher thermal resistance with a degradation initiated around $250{ }^{\circ} \mathrm{C}$, but after the TEMPO-oxidation this difference had vanished. Note that in Fig. 1 (and in the following) "TO" denotes the TEMPO-oxidized samples.

\section{Conductometric titration}

The summarised result from the conductometric titration of the different suspensions is given in Table 1 in terms of the carboxylate content. Before the TEMPOmediated oxidation the carboxylate content of the BE$\mathrm{CNC}$ was quite low. The unmodified $\mathrm{CNC}$ was subjected to a desulfonation step after the acid treatment and a rather small amount of sulphate groups remained on the surfaces after this treatment (corresponding to a z-potential of approximately $-35 \mathrm{mV}$ at $\mathrm{pH}$ 5.8). After the TEMPO-oxidation the carboxylate content was about the same for all the three different nanocellulosics and their surface charge in an aqueous
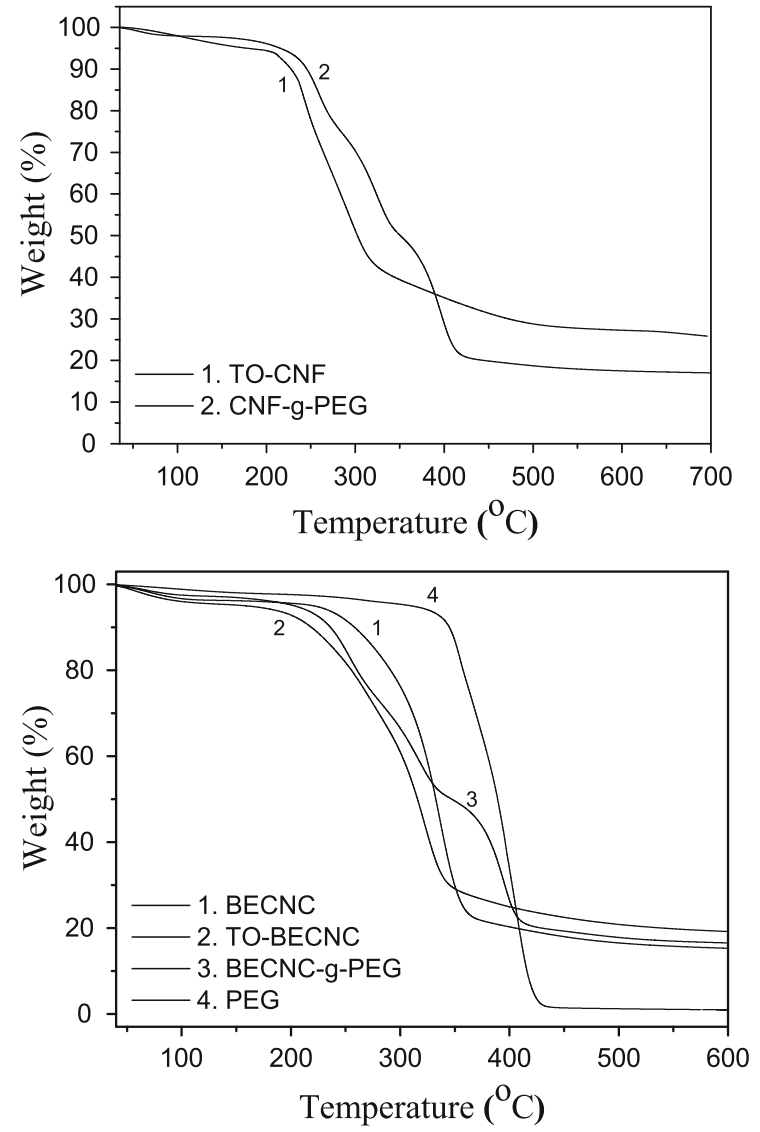

Fig. 1 Thermogravimetric curves showing the thermal degradation of PEG, TO-CNF and BE-CNC (including their modifications)

suspension is then expected to be similar. The PEGgrafting reduced the carboxylate content, which is to be expected since the amino-terminated PEG binds to the carboxylate groups during the grafting. Of the grafted nanomaterials, the $\mathrm{CNC}$ exhibited the lowest carboxylate content $\left(0.3 \mathrm{mmol} \mathrm{g}^{-1}\right)$.

\section{Fourier transform infrared spectroscopy (FTIR)}

Fourier transform IR spectroscopy can be used to follow the TEMPO-oxidation and the subsequent grafting of PEG onto the cellulose surfaces. Figure 2 constitutes an example of this in the case of the BECNC samples; similar results applied also to TO-CNF and BNC, cf (Tang et al. 2015).

For the TEMPO-oxidized sample, a new band at $1729 \mathrm{~cm}^{-1}$ appeared (compared to the unmodified $\mathrm{BE}-\mathrm{CNC}$ ) which could be associated with $\mathrm{C}=\mathrm{O}$ 
Table 1 Carboxylate content of the different samples before and after the grafting procedure

\begin{tabular}{ll}
\hline Nanocellulose & Carboxylate content $\left(\mathrm{mmol} \mathrm{g}^{-1}\right)$ \\
\hline TO-CNF & 1.2 \\
CNF-g-PEG & 0.43 \\
BE-CNC & 0.07 \\
TO-BE-CNC & 1.2 \\
BE-CNC-g-PEG & 0.34 \\
TO-CNC & 1.2 \\
CNC-g-PEG & 0.3 \\
\hline
\end{tabular}

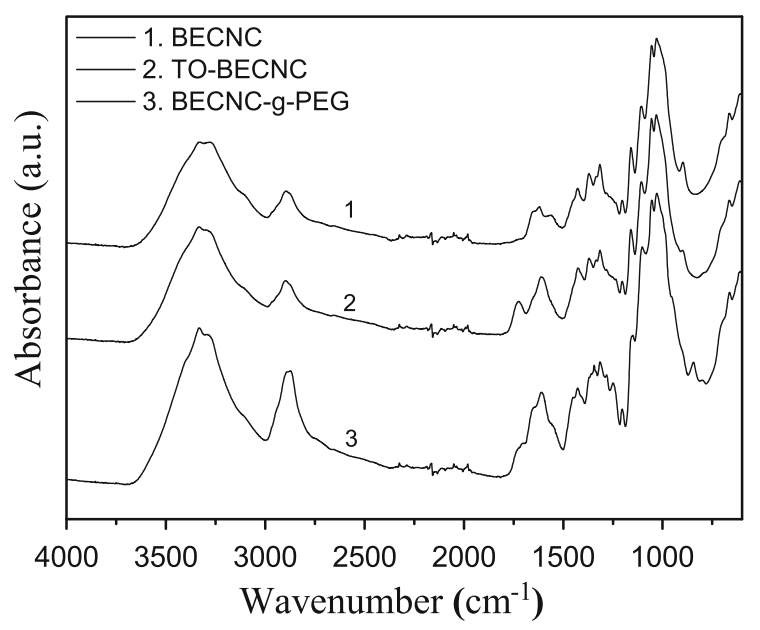

Fig. 2 Fourier transform IR spectra of the BE-CNC as received, TEMPO-oxidised and grafted with PEG

stretching frequency of the carbonyl group in the acidic form (Tang et al. 2015). The intensity of this peak decreased in the spectrum for the grafted sample which is expected since these groups were used for the grafting of PEG. Instead new bands appeared at 1558 and $1659 \mathrm{~cm}^{-1}$, associated with $\mathrm{NH}$ bending and carbonyl stretching, respectively, indicating a successful grafting of the amino-terminated PEG [these bands were however partially hidden since they overlap with absorption band of water associated with cellulose as remarked by Tang et al. (2015)].

The dimensions of the cellulosic elements

The different samples were imaged by both TEM and AFM in order to estimate the dimensions, i.e. the length and the diameter, of the cellulosic elements and how (and if) these were affected by the TEMPOoxidation and the subsequent grafting. Figure 3 is a

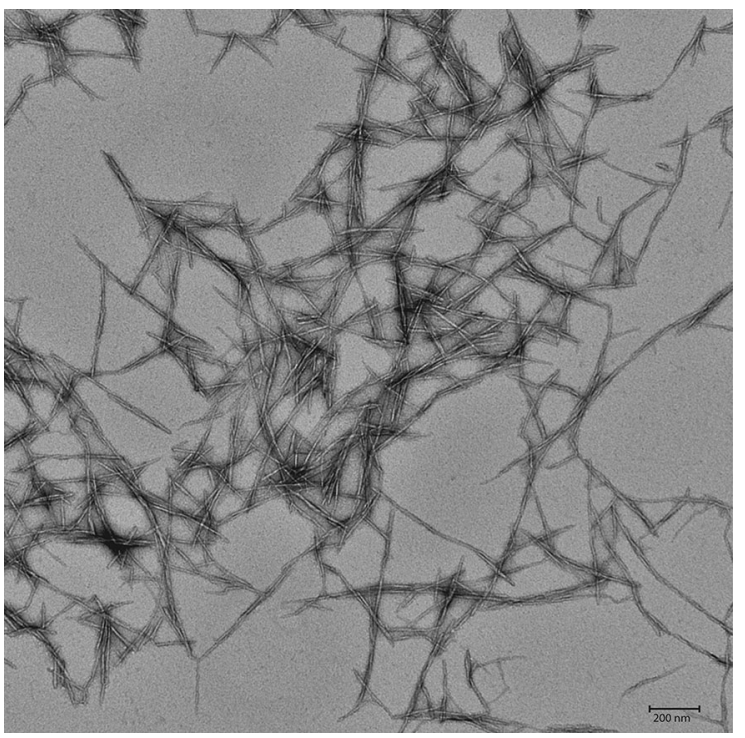

Fig. 3 Transmission electron micrograph of BE-CNC-g-PEG, stained with uranyl acetate in order to increase the contrast against the carbon grid on which the suspension was deposited and dried

TEM micrograph of a sample of BE-CNC-g-PEG. Individual "fibrils" can clearly be detected and a rough estimate of the length and diameter is 500 and $10 \mathrm{~nm}$, respectively, giving an aspect ratio (the ratio between length and diameter) of around 50. The dimensions will however be more commented on in the following.

Figure 4 shows AFM micrographs of a number of different nanocellulosics used in the present work. When determining the diameter of the elements it has already been mentioned that it is better to determine the height from measurements of this kind and then identifying the height with the diameter of the entities. This is associated with the size of the measuring tip in relation to the size of the object. AFM micrographs can be obtained with different imaging modes. Figure 4 contains examples of both the topographic mode (the contrast is based on height differences) and the phase mode (contrast depends on the stiffness of the material and the adhesion between the measuring tip and the specimen).

From the AFM images in Fig. 4, it seems like TOCNF consists of the longest elements, which is quite plausible, whereas the BE-CNC are somewhat shorter and also thicker. CNC apparently contains the shortest entities, which also is to be expected. It is also evident 

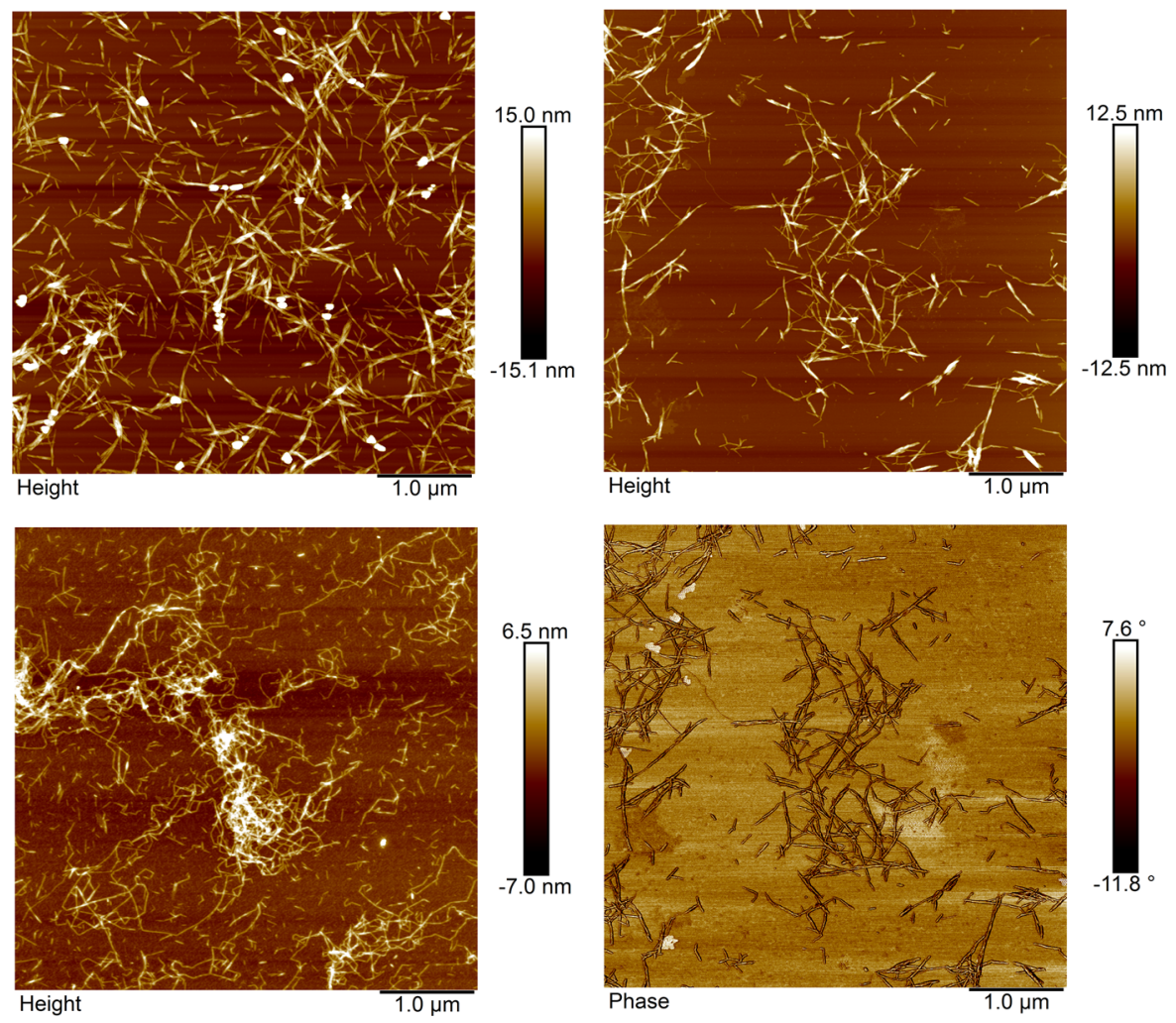

Fig. 4 AFM micrographs of three different samples. Top left shows a CNC sample, top right $\mathrm{BE}-\mathrm{CNC}$, bottom left TO-CNF, the bottom right shows a BE-CNC sample obtained in the phase mode. The other three images were produced with the topographic mode

that the CNF suspension contained several bent elements in agreement with earlier reports, e.g. (Usov and Mezzenga 2015). As outlined in the section on Characterization methods, the average values of the length and diameter of the different elements could be determined from the TEM and AFM micrographs together with their corresponding standard deviations. The results are summarized in Table 2 below.

It is obvious that the TO-CNF fibrils are the longest, followed by the BE-CNC elements and the CNC contains the shortest entities. It is also evident that the grafting of the PEG in most cases increased the diameter of the elements somewhat (the change is however not substantial), whereas neither the TEMPOoxidation nor the grafting had any significant effect on the length of the elements. The standard deviations are quite substantial, indicating that the size distributions of the elements are broad. In the case of the CNC and its modifications, this means that the suspensions most likely contained a relatively large number of particles that are small with a low aspect ratio.
Table 2 The average diameter and the average length of the nanocellulosic elements (together with the corresponding standard deviations) obtained from TEM or AFM images of the samples

\begin{tabular}{lll}
\hline Nanocellulose & Diameter $(\mathrm{nm})$ & Length $(\mathrm{nm})$ \\
\hline TO-CNF $^{1}$ & $3.6 \pm 0.7$ & $820 \pm 570$ \\
CNF-g-PEG $^{1}$ & $4.3 \pm 0.6$ & $830 \pm 480$ \\
BE-CNC $^{1}$ & $5.6 \pm 1.0$ & $520 \pm 310$ \\
TO-BE-CNC $^{1}$ & $5.7 \pm 1.2$ & $570 \pm 280$ \\
BE-CNC-g-PEG $^{1}$ & $6.3 \pm 1.4$ & $520 \pm 280$ \\
CNC $^{2}$ & $6.0 \pm 1.5$ & $211 \pm 114$ \\
TO-CNC $^{2}$ & $5.5 \pm 2.6$ & $198 \pm 105$ \\
CNC-g-PEG $^{2}$ & $5.2 \pm 1.4$ & $190 \pm 97$ \\
\hline
\end{tabular}

1 From TEM and ${ }^{2}$ from AFM

Comparisons with dimensions of $\mathrm{CNF} / \mathrm{CNC}$ reported elsewhere are not straightforward, since those depend on the raw material and the preparation technique (and to some extent also on the method used for evaluating the dimensions). However, it can be 
noted that Isogai et al. (2011) reported lengths of a few micrometers and widths of 3-4 $\mathrm{nm}$ for CNF obtained through TEMPO-mediated oxidation, whereas Wågberg et al. (2008) estimated the length of CNF produced via carboxymethylation to be up to $1000 \mathrm{~nm}$ and the width being in the range 5-15 nm. These reported values of the width were thus of the same order as those obtained here, whereas the length of the CNF used in the present work appears to be somewhat shorter, at least when comparing to Isogai et al. (2011). Shafiei-Sabet et al. (2013) used transmission electron microscopy to estimate the length and width of their CNC particles and reported values of about 100 and $7 \mathrm{~nm}$, respectively. These values are in reasonable agreement with the ones given in Table 2 above. The difference in length could possibly be attributed to that different fibre sources where used for the preparation of the CNC.

\section{Rheological properties}

The rheological properties were measured in shear for all the different samples at a temperature of $25^{\circ} \mathrm{C}$. The concentrations of the suspensions were 1 and $2 \mathrm{wt} \%$ (or $1.5 \mathrm{wt} \%$ ) for the $\mathrm{CNC}$ and the BE-CNC samples, whereas it was kept at $0.7 \mathrm{wt} \%$ in the case of the CNF suspension due to its significantly higher viscosity (at 1 and $2 \mathrm{wt} \%$ ) compared to that of the two other samples. Figure 5 shows the shear viscosity as a function of the shear rate in case of the lower concentrations of the suspensions ( 0.7 and $1 \mathrm{wt} \%)$.

The viscosity at low shear rates was highest for the $\mathrm{CNF}$ samples and of the same magnitude for the unmodified BE-CNC, being more than 10,000 mPas. Again, it should be pointed to that the concentration of the CNF suspensions was lower than that of the BECNC. All the unmodified CNC-materials and both CNF samples also exhibited a shear-thinning behaviour, which is the expected behaviour for suspensions containing rod-like particles or fibrils since these tend to orient themselves and partly disentangle due to the flow leading to a lower viscosity as the shear rate increases.

The viscosities of the TEMPO-oxidised TO-BE$\mathrm{CNC}$ and the unmodified $\mathrm{CNC}$, were significantly lower than that of the CNFs and the unmodified BE$\mathrm{CNC}$, being less than $100 \mathrm{mPas}$ over the shear rate region used here. The three other suspensions, the grafted BE-CNC, grafted $\mathrm{CNC}$ and TO-CNC,

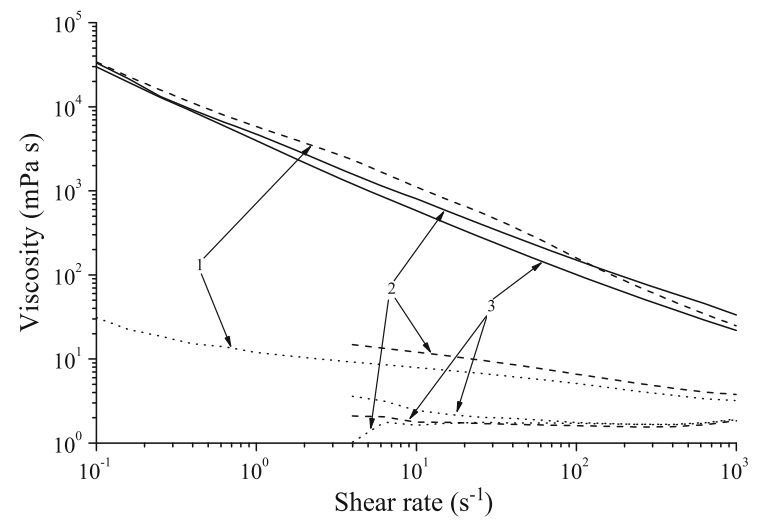

Fig. 5 The viscosity as a function of the shear rate for all the different sample at $1 \mathrm{wt} \%$ (0.7 wt \% for CNF Solid lines denote $\mathrm{CNF}$ samples, dashed lines $\mathrm{BE}-\mathrm{CNC}$ and dotted lines $\mathrm{CNC}, 1$ denotes unmodified specimens, 2 TEMPO-oxidised specimens and 3 the PEG-grafted specimens

displayed even lower viscosities and reliable measurements could only be performed at shear rates exceeding approximately $5 \mathrm{~s}^{-1}$. These latter suspensions exhibited an almost Newtonian behaviour with a viscosity of about $2 \mathrm{mPas}$.

Figure 6 summarizes the shear rate dependence of the viscosity at a higher concentration of some of the nanocellulosics $(2 \mathrm{wt} \%$ in case of unmodified BE$\mathrm{CNC}$ and $\mathrm{CNC}$, TO-CNC, grafted $\mathrm{BE}-\mathrm{CNC}$ and $1.5 \mathrm{wt} \%$ for TO-BE-CNC). As expected, the increase in concentration resulted in a higher viscosity, $\mathrm{cf}$ e.g. (Barnes et al. 1989). The same pattern of behaviour as evident at the lower concentration was also at hand when the concentration was increased. The viscosity level of TO-CNC, grafted CNC and grafted BE-CNC was somewhat increased, but the measured values were still very low.

An important conclusion from this work is that the rheological properties of the nanocellulose dispersions depend significantly on the manufacturing technique and thus the dimensions and surface character of the particles. This in itself points to difficulties in comparing the results obtained here to those reported in the literature. However, it may be mentioned that ShafieiSabet et al. (2013) measured the shear viscosity of $\mathrm{CNC}$ dispersions as a function of the shear rate. At a concentration of $1 \mathrm{wt} \%$ and shear rates between 0.1 and $1 \mathrm{~s}^{-1}$, the measured viscosity was found to be in the region $10-100 \mathrm{mPas}$, which is not too different from the results shown in Fig. 5, despite that the dimensions and surface character of the nanoparticles 


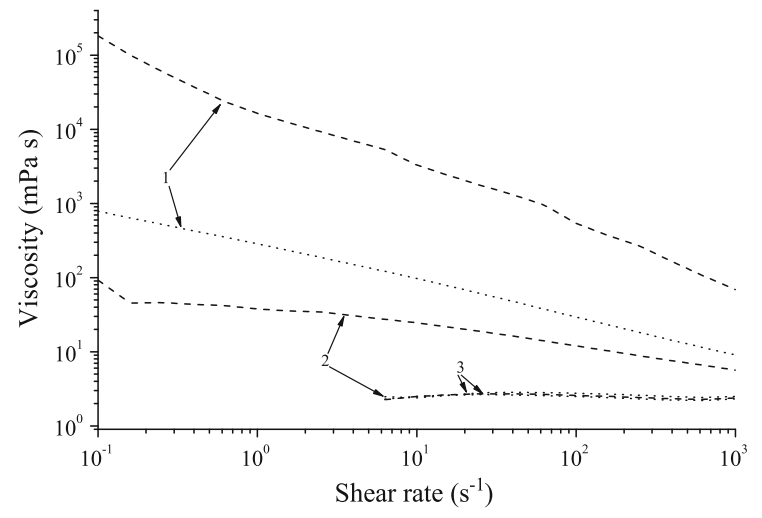

Fig. 6 Same as Fig. 5 but at a higher concentration of the nanocellulosics in the suspensions $(2 \mathrm{wt} \%$ in case of unmodified $\mathrm{BE}-\mathrm{CNC}$ and $\mathrm{CNC}$, TO-CNC, grafted BE-CNC, grafted CNC, and $1.5 \mathrm{wt} \%$ for TO-BE-CNC). Dashed lines denote BE-CNC and dotted lines $\mathrm{CNC}, 1$ denotes unmodified specimens, 2 TEMPO-oxidised specimens and 3 the PEG-grafted specimens

are expected to differ. Naderi et al. (2014) evaluated the shear viscosity of carboxymethylated CNF suspensions at shear rates ranging from 0.1 to $1000 \mathrm{~s}^{-1}$ and noted viscosity values decreasing from about $10^{6}-$ $10 \mathrm{mPas}$. The CNF-concentration was in this case $0.6 \mathrm{wt} \%$. The viscosity levels were in the same range as obtained here at higher shear rates (Fig. 5), but in the low shear rate region, lower viscosity values were noted in the present work.

In this context it may be of interest to estimate at which concentration a percolating network is formed in the suspension, since the formation of such a network is expected to have strong influence on the rheological behaviour, see e.g. (Kotsilkova 2007; Kim and Macosko 2009; Oxfall et al. 2015). In order to predict the onset of percolation $\left(\varphi_{c}\right)$ in terms of a volume concentration, (Xu et al. 2013) used

$\varphi_{c}=0.7 \frac{w}{L}$ where $w$ is the width (diameter) of the elements and $L$ their length. Then numerical value 0.7 can be discussed, e.g. (Hill 2008) suggested a somewhat higher value, and possible effects of flow-induced orientation and particle interactions are discarded from here. With these reservations and admitting the large standard deviations in the measured dimensions, the average length and diameter could be used in order to at least estimate $\varphi_{c}$. Table 3 gives the calculated values for the different suspensions expressed in vol\%. The concentration of the nanocellulosic suspensions is also given assuming a density of cellulose of $1.5 \mathrm{~g} \mathrm{~cm}^{-3}$ and water of $1.0 \mathrm{~g} \mathrm{~cm}^{-3}$.

Here it may be noted that the concentration of the CNF-based suspension clearly exceeded the critical percolation concentration, which is also reflected in the relatively high viscosity of these suspensions. In case of the BE-CNC suspensions, the concentrations were in the same range as $\varphi_{c}$, whereas with the $\mathrm{CNC}$ the percolation limit seemed to exceed the concentration of the suspensions. It should be clear, for reasons already stated, that the exact value of $\varphi_{c}$ should not be taken too strictly but merely used as an indication towards interaction between the cellulosic elements and thus a reason for increased viscosity.

All suspensions were also subjected to an oscillating shear deformation with a successively increased strain amplitude at $1 \mathrm{~Hz}$ and the shear storage $\left(G^{\prime}\right)$ and loss $\left(G^{\prime \prime}\right)$ moduli were then evaluated as functions of this amplitude. Typical examples of the results obtained are shown in Fig. 7 in the case of CNF $(0.7 \mathrm{wt} \%)$ and BE-CNC ( $1 \mathrm{wt} \%)$. The results mirror in a sense the measured shear viscosity, i.e. higher moduli are in general reflected in a higher shear viscosity. Both types of CNF and the TO-BE-CNC exhibit quite high values of the storage and loss moduli and they are approximately of the same order for all
Table 3 The weight and volume concentrations of the different suspensions and the critical volume concentration $\left(\varphi_{c}\right)$ for the onset of percolation

\begin{tabular}{llll}
\hline Nanocellulose & Concentration $(\mathrm{wt} \%)$ & Concentration $(\mathrm{vol} \%)$ & $\varphi_{c}(\mathrm{vol} \%)$ \\
\hline TO-CNF & 0.7 & 0.47 & 0.31 \\
CNF-g-PEG & 0.7 & 0.47 & 0.36 \\
BE-CNC & $1.0 ; 2.0$ & $0.67 ; 1.34$ & 0.75 \\
TO-BE-CNC & $1.0 ;$ & $0.67 ;$ & 0.70 \\
BE-CNC-g-PEG & $1.0 ; 2.0$ & $0.67,1.34$ & 0.85 \\
CNC & $1.0 ; 2.0$ & $0.67 ; 1.34$ & 1.99 \\
TO-CNC & $1.0 ; 2.0$ & $0.67 ; 1.34$ & 1.94 \\
CNC-g-PEG & $1.0 ;$ & $0.67 ;$ & 1.92 \\
\hline
\end{tabular}



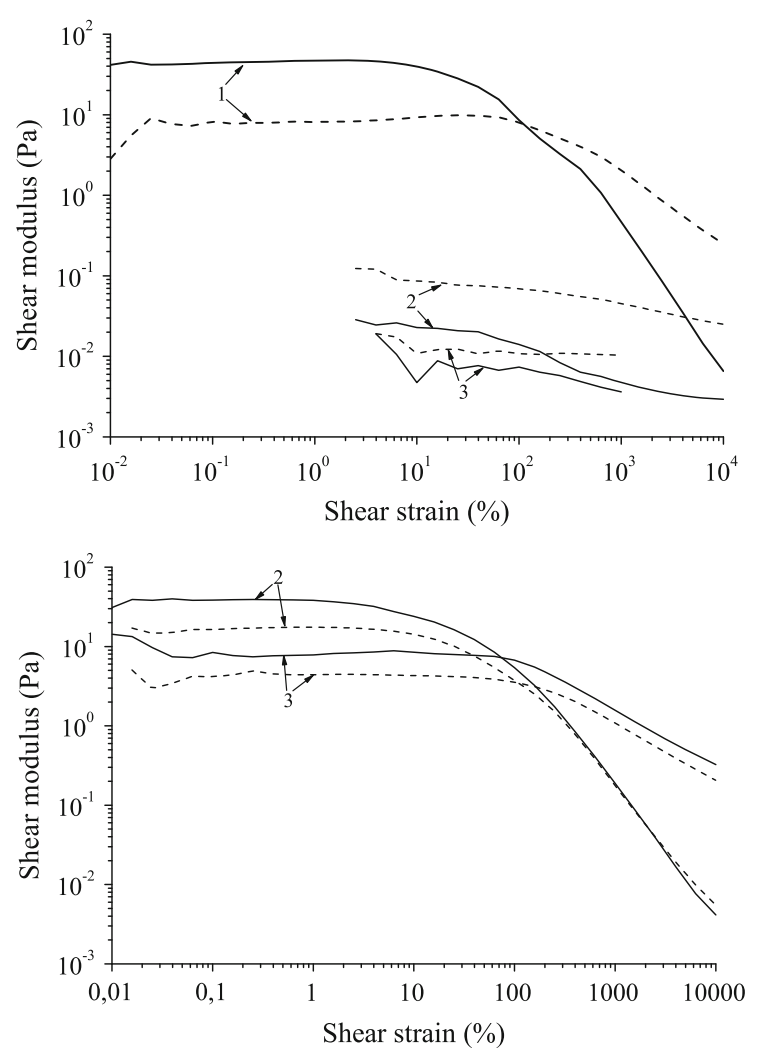

Fig. 7 Dynamic strain sweep of the BE-CNC samples (upper graph, $1 \mathrm{wt} \%$ ) and the CNF samples (lower graph, $0.7 \mathrm{wt} \%$ ). $\mathrm{G}^{\prime}$ and $\mathrm{G}^{\prime \prime}$ are shown as function of the shear strain amplitude at a frequency of $1 \mathrm{~Hz} ; 1$ denotes the unmodified BE-CNC, 2 the tempo-oxidised samples and 3 the grafted specimens Solid lines denote the storage modulus and dashed lines the loss modulus

the three suspensions. (Note however that the CNF concentration is lower than that of the BE-CNC suspensions.) In the linear viscoelastic region, i.e. where the moduli are independent of the applied shear strain, $G^{\prime}$ is higher than $G^{\prime \prime}$, which indicates a solid- or gel-like behaviour. At a certain "critical" strain amplitude, which was almost the same for all these suspensions, both the moduli started to decrease, in agreement with earlier observations (Moberg and Rigdahl 2012; Naderi et al. 2014). This would correspond to a breakdown or disruption of the "elastic" network formed by the cellulosic elements. Grafting of PEG to the cellulose surfaces reduced the storage and the loss moduli for both types of suspensions. A contributing reason for this decrease could be that in the grafted systems, the cellulose content is actually lower, since fibres/particles are grafted with more than $30 \mathrm{wt} \%$ PEG.
Both the unmodified and the grafted BE-CNC displayed significantly lower values of $G^{\prime}$ and $G^{\prime \prime}$ than the suspensions discussed above. For the former suspensions, the loss modulus was also greater than the storage modulus in the shear strain region covered. This would indicate a more liquid-like behaviour and there was no clear sign of any network formation in these specimens (as reflected in the dynamic mechanical analysis). For these BE-CNC suspensions reliable measurements could only be obtained using strain amplitude greater than approximately $10 \%$ (the torque readings were too low and scattered in the low strain region). For similar reasons, in case of the CNC-based suspensions, their viscoelastic properties were too low to be measured with any reliability.

\section{Discussion and conclusions}

The FTIR and the TGA results showed that the TEMPO-oxidation and the PEG-grafting onto the cellulose surfaces were successful. The TGA was also used for assessing the amount of grafted material onto the cellulosic entities, which turned out to be rather similar for the three types of cellulosic elements. After the TEMPO-oxidation, the carboxylate content was similar for the three types of nanocelluloses used here, and the PEG-grafting reduced, as expected, the carboxylate content, which were not too different for the three types of samples, cf Table 1 . The changes brought about when changing the surface character was certainly expected to affect the rheological properties of the corresponding suspensions.

Turning now to the viscosity of the suspensions containing the TEMPO-oxidized fibrils and rods, it is evident the viscosity level is influenced by the length (or aspect ratio) of the elements or could be in a sense be associated with the critical volume content for the onset of percolation $\varphi_{c}$. The TO-CNF suspensions exhibited the highest viscosity (and the concentration was clearly higher than $\varphi_{c}$ ) followed by TO-BE-CNC which displayed a lower aspect ratio. The lowest viscosity was noted for the TO-CNC and in this case the concentrations used were lower than $\varphi_{c}$ which indicates that the no percolating network was formed in the suspension. This is also supported by the viscoelastic results, i.e. very low values of the moduli were noted. The presence of a fibrillar network in the TO-CNF suspensions which was broken down at 
larger deformations was quite evident in the dynamicmechanical analysis. It may be underlined once again that some caution of taking the values of $\varphi_{c}$ to literally; they are subjected to large variations and in principle they do not account for interactions between the elements. It is well known that the percolation threshold can be affected by particle interactions, cf e.g. (Safran et al. 1985) and thus Eq. (1) should only be regarded as an approximation providing a qualitative indication of the percolation behaviour. A detailed account of the influence of the surface characteristics on the onset of percolation of the nanocellulose particles is however beyond the scope of this study.

Prior to the TEMPO-oxidation, the surface charge of the cellulosic materials was significantly lower, see Table 1. The electrostatic repulsion associated with the surface charge retards aggregation/mechanical entanglement and floc/network formation and would thus result in lower viscosity levels. As can be seen in Fig. 5, the BE-CNC and CNC suspensions which were not TEMPO-oxidised clearly exhibited a higher viscosity than their TEMPO-oxidized counterparts. This cannot be related to the geometry of the cellulosic elements since their dimensions were more or less unaffected by the oxidation, Table 2 . Of the two, the BE-CNC suspension still exhibited the highest viscosity due its higher aspect ratio. The dynamicmechanical measurements clearly show that a network structure is formed with these low-charged BE-CNC elements.

Grafting of PEG onto the TO-CNF resulted only in a minor decrease of the viscosity and the viscoelastic properties of the suspension. An interpretation, in addition to the already mentioned reduction in the actual cellulose content, may be that the rheological response is mainly determined by the fibril network formed by the relatively long fibrils and the surface characteristics then play a secondary role here. The suspensions based on TO-CNC had a very low viscosity (on the border of being measureable) and the grafting of PEG to these particles had no greater influence on the rheological properties. In case of the TO-BE-CNC, however, the grafting resulted in a significant reduction of the viscosity, Figs. 5 and 6, despite the lowering of the carboxylate content. This was perhaps somewhat unexpected and points to other types of interactions, e.g. steric, contributing to the observed behaviour. Alternatively, the different treatments used here may possible have broken down parts of the cellulosic elements to very small and almost spherical particles which were not detected during the assessment of the dimensions of the particles. Further studies are however required in order to clarify the relevant mechanisms.

In conclusion, $\mathrm{CNF}$ and $\mathrm{CNC}$ materials and suspensions are not a unique class of substances exhibiting properties within a certain region. Here, it has been shown that in addition to the concentration of the fibrils/particles, the dimensions and surface character of the cellulosic elements can affect and control their rheological behaviour within a broad range. The aspect ratio of the particles plays a very important role here. This points to the importance of a careful characterisation of dimensions and surface character of the elements when comparing such systems and using them in different situations.

Acknowledgments The authors thank Wallenberg Wood Science Center and Chalmers University of Technology for the financial support.

Open Access This article is distributed under the terms of the Creative Commons Attribution 4.0 International License (http:// creativecommons.org/licenses/by/4.0/), which permits unrestricted use, distribution, and reproduction in any medium, provided you give appropriate credit to the original author(s) and the source, provide a link to the Creative Commons license, and indicate if changes were made.

\section{References}

Barnes HA, Hutton JF, Walters K (1989) An introduction to rheology, Ch. 7. Elsevier, Amsterdam

Eichhorn SJ, Dufresne A, Aranguren M et al (2009) Review: current international research into cellulose nanofibres and nanocomposites. J Mater Sci 45:1-33. doi:10.1007/ s10853-009-3874-0

Hasani M, Cranston ED, Westman G, Gray DG (2008) Cationic surface functionalization of cellulose nanocrystals. Soft Matter 4:2238-2244. doi:10.1039/B806789A

Henriksson M, Henriksson G, Berglund LA, Lindström T (2007) An environmentally friendly method for enzyme-assisted preparation of microfibrillated cellulose (MFC) nanofibers. Eur Polym J 43:3434-3441. doi:10.1016/j.eurpolymj. 2007.05.038

Hill RJ (2008) Elastic modulus of microfibrillar cellulose gels. Biomacromol 9:2963-2966. doi:10.1021/bm800490x

Hooshmand S, Aitomäki Y, Skrifvars M et al (2014) All-cellulose nanocomposite fibers produced by melt spinning cellulose acetate butyrate and cellulose nanocrystals. Cellulose 21:2665-2678. doi:10.1007/s10570-014-0269-4

Iotti M, Gregersen ØW, Moe S, Lenes M (2010) Rheological studies of microfibrillar cellulose water dispersions. 
J Polym Environ 19:137-145. doi:10.1007/s10924-0100248-2

Isogai A, Saito T, Fukuzumi H (2011) TEMPO-oxidized cellulose nanofibers. Nanoscale 3:71-85. doi:10.1039/CONR 00583E

Jiang F, Esker AR, Roman M (2010) Acid-catalyzed and solvolytic desulfation of $\mathrm{H}_{2} \mathrm{SO}_{4}$-hydrolyzed cellulose nanocrystals. Langmuir 26:17919-17925. doi:10.1021/ la1028405

Kantor TG, Schubert M (1957) The difference in permeability of cartilage to cationic and anionic dyes. J Histochem Cytochem 5:28-32. doi:10.1177/5.1.28

Karppinen A, Vesterinen A-H, Saarinen T et al (2011) Effect of cationic polymethacrylates on the rheology and flocculation of microfibrillated cellulose. Cellulose 18:1381-1390. doi:10.1007/s10570-011-9597-9

Kim H, Macosko CW (2009) Processing-property relationships of polycarbonate/graphene composites. Polymer 50: 3797-3809. doi:10.1016/j.polymer.2009.05.038

Kotsilkova R (ed) (2007) Thermoset nanocomposites for engineering applications. Smithers Rapra Technology, Shrewsbury

Lee K-Y, Aitomäki Y, Berglund LA et al (2014) On the use of nanocellulose as reinforcement in polymer matrix composites. Compos Sci Technol 105:15-27. doi:10.1016/j. compscitech.2014.08.032

Mathew AP, Oksman K, Karim Z et al (2014) Process scale up and characterization of wood cellulose nanocrystals hydrolysed using bioethanol pilot plant. Ind Crops Prod 58:212-219. doi:10.1016/j.indcrop.2014.04.035

Moberg T, Rigdahl M (2012) On the viscoelastic properties of microfibrillated cellulose (MFC) suspensions. Annu Trans Nord Rheol Soc 20:123-130

Moberg T, Rigdahl M, Stading M, Levenstam Bragd E (2014) Extensional viscosity of microfibrillated cellulose suspensions. Carbohydr Polym 102:409-412. doi:10.1016/j. carbpol.2013.11.041

Moberg T, Tang H, Zhou Q, Rigdahl M (2016) Preparation and viscoelastic properties of composite fibres containing cellulose nanofibrils: formation of a coherent fibrillar network. J Nanomater 2016:e9569236. doi:10.1155/2016/ 9569236

Mongondry P, Bonnans-Plaisance C, Jean M, Tassin JF (2003) Mild synthesis of amino-poly(ethylene glycol)s. Application to steric stabilization of clays. Macromol Rapid Commun 24:681-685. doi:10.1002/marc.200350012

Naderi A, Lindström T (2016) A comparative study of the rheological properties of three different nanofibrillated cellulose systems. Nordic Pulp Pap Res J 31:364-371. doi:10.3183/NPPRJ-2016-31-03-p364-371

Naderi A, Lindström T, Sundström J (2014) Carboxymethylated nanofibrillated cellulose: rheological studies. Cellulose 21:1561-1571. doi:10.1007/s10570-014-0192-8

Oxfall H, Ariu G, Gkourmpis T et al (2015) Effect of carbon black on electrical and rheological properties of graphite nanoplatelets/poly(ethylene-butyl acrylate) composites. Expr Polym Lett 9:66-76. doi:10.3144/expresspolymlett.2015.7

Pääkkö M, Ankerfors M, Kosonen H et al (2007) Enzymatic hydrolysis combined with mechanical shearing and highpressure homogenization for nanoscale cellulose fibrils and strong gels. Biomacromol 8:1934-1941. doi:10.1021/bm $061215 p$

Safran SA, Webman I, Grest GS (1985) Percolation in interacting colloids. Phys Rev A 32:506-511. doi:10.1103/ PhysRevA.32.506

Sakurada I, Nukushina Y, Ito T (1962) Experimental determination of the elastic modulus of crystalline regions in oriented polymers. J Polym Sci 57:651-660. doi:10.1002/pol. 1962.1205716551

Shafiei-Sabet S, Hama WY, Hatzikiriakos SG (2013) Influence of the degree of sulfation on the rheology of cellulose nanocrystal suspensions. Rheol Acta 52:741-751. doi:10. 1007/s00397-013-0722-0

Tang H, Butchosa N, Zhou Q (2015) A transparent, hazy, and strong macroscopic ribbon of oriented cellulose nanofibrils bearing poly(ethylene glycol). Adv Mater 27:2070-2076. doi:10.1002/adma.201404565

Turbak AF, Snyder FW, Sandberg KRJ (1983) Microfibrillated cellulose, a new cellulose product: properties, uses and commercial potential. J Appl Polym Sci 37:815-827

Usov I, Mezzenga R (2015) FiberApp: an open-source software for tracking and analyzing polymers, filaments, biomacromolecules, and fibrous objects. Macromolecules 48:1269-1280. doi:10.1021/ma502264c

Wågberg L, Decher G, Norgren M et al (2008) The build-up of polyelectrolyte multilayers of microfibrillated cellulose and cationic polyelectrolytes. Langmuir 24:784-795. doi:10.1021/la702481v

Wang Q, Zhu JY (2016) Effects of mechanical fibrillation time by disk grinding on the properties of nanocellulose fibrils. Tappi J 15:419-423

Xu X, Liu F, Jiang L et al (2013) Cellulose nanocrystals vs. cellulose nanofibrils: a comparative study on their microstructures and effects as polymer reinforcing agents. ACS Appl Mater Interfaces 5:2999-3009. doi:10.1021/ am302624t 